\title{
Development of a standardized mucositis and osteoradionecrosis animal model using external radiation
}

\author{
Mi Hyun Seo ${ }^{1}$, Min Young Lee ${ }^{3}$, Mi Young Eo', Suk Keun Lee ${ }^{4}$, Kyung Mi Woo², Soung Min Kim \\ ${ }^{l}$ Department of Oral and Maxillofacial Surgery, ${ }^{2}$ Department of Pharmacology \& Dental Therapeutics, \\ Dental Research Institute, School of Dentistry, Seoul National University, Seoul, \\ ${ }^{3}$ Laboratory Animal Center, Korea Institute of Radiological and Medical Sciences, Seoul, \\ ${ }^{4}$ Department of Oral Pathology, College of Dentistry, Gangneung-Wonju National University, Gangneung, Korea
}

\begin{abstract}
J Korean Assoc Oral Maxillofac Surg 2020;46:240-249)
Objectives: Although the side effects of radiation therapy vary from mucositis to osteomyelitis depending on the dose of radiation therapy, to date, an experimental animal model has not yet been proposed. The aim of this study was to develop an animal model for assessing complications of irradiated bone, especially to quantify the dose of radiation needed to develop a rat model.

Materials and Methods: Sixteen Sprague-Dawley rats aged seven weeks with a mean weight of $267.59 \mathrm{~g}$ were used. Atraumatic extraction of a right mandibular first molar was performed. At one week after the extraction, the rats were randomized into four groups and received a single dose of external radiation administered to the right lower jaw at a level of 14, 16, 18, or $20 \mathrm{~Gy}$, respectively. Clinical alopecia with body weight changes were compared and bony volumetric analysis with micro-computed tomography (CT), histologic analysis with H\&E were performed.

Results: The progression of the skin alopecia was different depending on the irradiation dose. Micro-CT parameters including bone volume, bone volume/tissue volume, bone mineral density, and trabecular spaces, showed no significant differences. The progression of osteoradionecrosis (ORN) along with that of inflammation, fibrosis, and bone resorption, was found with increased osteoclast or fibrosis in the radiated group. As the radiation dose increases, osteoclast numbers begin to decrease and osteoclast tends to increase. Osteoclasts respond more sensitively to the radiation dose, and osteoblasts are degraded at doses above $18 \mathrm{~Gy}$.

Conclusion: A standardized animal model clinically comparable to ORN of the jaw is a valuable tool that can be used to examine the pathophysiology of the disease and trial any potential treatment modalities. We present a methodology for the use of an experimental rat model that incorporates a guideline regarding radiation dose.
\end{abstract}

Key words: Osteoradionecrosis of jaw, External radiation therapy, Experimental animal model, Mucositis, Osteoclast

[paper submitted 2019. 8. 9 / revised 2019. 9. 13 / accepted 2019. 9. 16]

\section{Kyung Mi Woo}

Department of Pharmacology \& Dental Therapeutics, Dental Research Institute, School of Dentistry, Seoul National University, 101 Daehak-ro, Jongno-gu, Seoul 03080, Korea

TEL: +82-2-740-8652 FAX: +82-2-741-3193

E-mail:kmwoo@snu.ac.kr

ORCID: https://orcid.org/0000-0002-2782-1189

\section{Soung Min Kim}

Department of Oral and Maxillofacial Surgery, Dental Research Institute, School of Dentistry, Seoul National University, 101 Daehak-ro, Jongnogu, Seoul 03080, Korea

TEL: +82-2-2072-0213 FAX: +82-2-766-4948

E-mail:smin5@snu.ac.kr

ORCID: https://orcid.org/0000-0002-6916-0489

(c) This is an open-access article distributed under the terms of the Creative Commons Attribution Non-Commercial License (http://creativecommons.org/ licenses/by-nc/4.0/), which permits unrestricted non-commercial use, distribution, and reproduction in any medium, provided the original work is properly cited.

Copyright (C) 2020 The Korean Association of Oral and Maxillofacial Surgeons. All rights reserved.

\section{Introduction}

Radiation therapy is an essential treatment method and one of the existing multidisciplinary treatments available for head and neck cancer. Notably, one significant adverse effect of radiotherapy is osteoradionecrosis (ORN) of the mandible ${ }^{1}$. ORN is a serious complication that occur after radiotherapy known to affect the mandible by $2 \%$ to $22 \%{ }^{2}$. ORN is a pathologic condition of the jaw based on clinical criteria, through the pathogenesis of this disease remains unclear. Currently, there are two main theories that explain the pathophysiology of ORN ${ }^{3}$. Marx ${ }^{4}$ suggested the suitability of the "3Hs" theory in 1983, in that radiation induces hypovascularity, causing hypoxia in the tissues and leading to cell death (i.e., hypocellularity). In contrast, Delanian and Lefaix ${ }^{5}$ presented the radiation-induced fibroatrophic (RIF) theory 
focusing on the molecular and cell biology perspectives in 2004. The RIF theory suggests that the main event for ORN progression is associated with the activation and deregulation of fibroblast activity and atrophic tissue formation at the irradiated site. The radiation may cause an imbalance between cell death and replacement and between collagen degradation and synthesis ${ }^{6}$. ORN can occur spontaneously; however, dental trauma, especially the extraction of teeth in a local radiated field can increase the incidence of ORN. ORN can be confirmed by clinical examination with the aid of histologic, radiologic assessments ${ }^{7}$. Commonly, ORN can be diagnosed based on clinical signs by observing (1) exposed bone without healing for between two and six months, (2) a history of radiation therapy performed in the region of the exposed bone, (3) the presence of necrotic or devitalized bone, and (4) no evidence of tumor recurrence ${ }^{8}$.

The change of histological structure of the mandibular bone was described in that it depended on the time and dose of irradiation. Cohen et al. ${ }^{7}$ suggested three weeks in an animal model of mandibular ORN as being analogous to three years in humans. The radiation decreases the regenerative capacity of bone ${ }^{9}$, making osteoblasts resistant to radiation damage and osteocytes sensitive to radiation. Osteoclasts can be damaged by irradiation with a single absorbed dose of 20 Gy. However, radiation inhibited the proliferation of osteoblasts and induced G2/M cell cycle arrest in vivo study ${ }^{10}$. Springer et al. ${ }^{11}$ described that, following irradiation, the osteoblasts and osteocytes were decreased histologically and hypocellular bony tissue was observed. However, the original bony structure itself was not disturbed.

There are studies that have detailed ORN canine ${ }^{12,13}$, rabbit $^{9}$, mouse ${ }^{14}$ and rat $^{15,16}$, animal models, respectively. However, these models were largely not optimal because of the dose of irradiation used in the studies: although the previous studies demonstrated mandibular bone damage after radiation treatment, there remains no consistent histopathological analysis and time of irradiation among them. Moreover, no translational aspect was demonstrated with regard to current radiation treatments for head and neck cancer in the clinical setting.

The goals of this study were to establish a clinically relevant model according to previous models of ORN; to explore the effective and optimal dose of radiation therapy; and to assess outcomes via H\&E staining, and micro-computed tomography (micro-CT). Additionally, we sought to develop a rat model of mandibular ORN, including quantifiable and standardized method with micro-CT analysis and histology.

\section{Materials and Methods}

\section{Animal preparation}

The present study's protocol was reviewed and approved by the Seoul National University Institutional Animal Care and Use Committee (SNU-121123-12-11). A total of 16 Sprague-Dawley male rats aged seven weeks with an average weight of $267.59 \mathrm{~g}$ (OrientBio, Seongnam, Korea) were housed in the Laboratory Animal Center of the Korea Institute of Radiological and Medical Sciences with a 12-hour light/dark cycle and were given chow and water ad libitum. The animals were housed for at least seven days prior to being used for this experiment. All of the experimental procedures were carried out in accordance with the "Recommendations for Handling of Laboratory Animals for Biomedical Research" complied by the Committee on the Safety and Ethical Handling Regulations for Laboratory Experiments at Seoul National University and Korea Institute of Radiological and Medical Sciences.

\section{Grouping and experimental design}

Extractions of teeth were performed under general anesthesia with an intraperitoneal injection of a mixture of Ketamin (90 mg/kg, ketamine hydrochloride; Yuhan, Seoul, Korea) and Rompun $(10 \mathrm{mg} / \mathrm{kg}$, xylazine; Bayer Korea, Seoul, Korea). The surgical procedure proceeded as follows: dermal and oral disinfection was completed with betadine, the mouth was opened, the gingiva around the right lower first molar was detached, and atraumatic extraction was performed. Under the general anesthesia, the animals were fixed in the supine position, with all extraction procedures gently performed with dental explorers and root forceps.

One week after the extraction, the extraction region was exposed to a graded single dose of radiation. In a previous study involving a mouse model, it was found that the histological damage healed at 14 days after both 14 Gy and 16 Gy of irradiation, while death occurred at nine days and 10 days after 18 Gy and 20 Gy of irradiation, respectively ${ }^{17}$. Modifications to a previously published rat model of mandibular $\mathrm{ORN}^{7}$ were made to develop an ideal protocol.

In the present study, 16 rats were divided into four groups. Next, a single dose of 14 Gy irradiation were applied to the right jaw in group 1 (four rats), a single dose of 16 Gy irradiation was applied to the right jaw in group 2 (four rats), a single dose of 18 Gy irradiation was applied to the right jaw 
in group 3 (four rats), and a single dose of 20 Gy irradiation was applied to the right jaw in group 4 (four rats), respectively. Another two rats were designated as the control group and did not undergo irradiation.

\section{Irradiation}

Radiation doses of 14, 16, 18, or 20 Gy were administered once to the right mandible of all experimental animals, with each animal under general anesthesia. We used the X-RAD 320 biological irradiator (Precision X-ray, North Branford, CT, USA), which has a $320-\mathrm{kVp}$ orthovoltage energy level with a $450 \mathrm{kV}$ maximum output (tube limited to $320 \mathrm{kV}$ ) and 45 maximum mA.(Fig. 1. A, 1. B) The field size was set at $10 \times 10 \mathrm{~mm}^{2}$, focusing on the right mandible, and the dose rate was $2.0 \mathrm{~Gy} / \mathrm{min}$. Irradiation time was 240 seconds in group 1, 480 seconds in group 2, 540 seconds in group 3, and 600 seconds in group 4, respectively.

\section{Animal sacrifice and clinical evaluation}

In each group, two animals were sacrificed two weeks after irradiation and the others were sacrificed three weeks after irradiation, respectively, via $\mathrm{CO}_{2}$ inhalation. The pathologic change of the bone and mucosa was investigated during the harvest of the mandible. The harvested mandibles were fixed in $10 \%$ formalin for micro-CT analysis. The weight of the rats was assessed in this study.(Table 1) Moreover, the clinical manifestations of irradiation observed on gross pathologic evaluation of rat mandibles before and after irradiation included alopecia of the facial skin, mucosa coverage of the wound, and denuded necrotic bone.

\section{Micro-CT analysis}

Micro-CT images was taken with the SkyScan 1172 microfocus X-ray system (Bruker, Kontich, Belgium). This device is equipped with a microfocus X-ray tube with a focal spot of $2 \mathrm{~mm}$ and produces a cone beam that is detected by a 12bit, cooled X-ray camera that was a charge-coupled device (CCD) fiber-optically coupled to a scintillator. The scan parameters were $70 \mathrm{kV}, 141 \mu \mathrm{A}, 0.5 \mathrm{~mm} \mathrm{Al}$ filter, and $360^{\circ}$ rotations with $0.4^{\circ}$ steps. A full three-dimensional (3D) image containing the experimental area and the surrounding trabecular bone was obtained with a cubic voxel size of $17 \mu \mathrm{m}$, resulting in about 900 slices 2D images per specimen.(Fig. 1. C) Image analysis was done with the CTAn 1.8 application (Bruker), while 3D reconstruction was done with NRecon 1.6.9.8 (Bruker). Using 3D reconstructed images, regions demonstrating consistent bony necrosis and bone formation
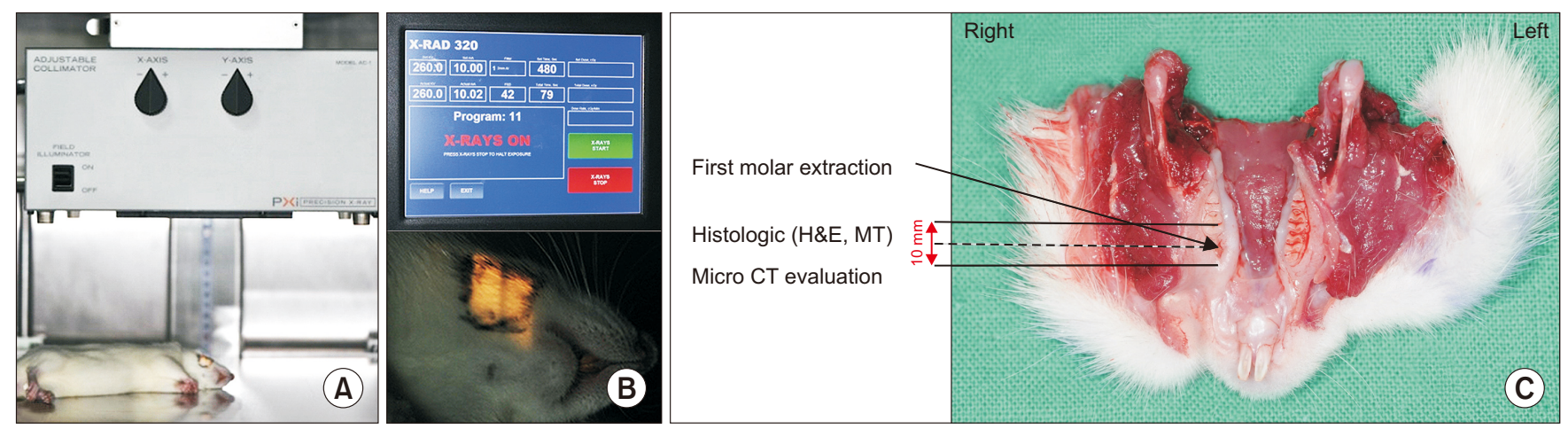

Fig. 1. A. Animal receiving irradiation. B. Experimental radiological setting used in this study, $260 \mathrm{kV}, 10 \mathrm{~mA}, 2 \mathrm{~mL}$ Al filter. C. Specimens for evaluation after harvesting of the lower jaw including soft tissue.

Mi Hyun Seo et al: Development of a standardized mucositis and osteoradionecrosis animal model using external radiation. J Korean Assoc Oral Maxillofac Surg 2020

Table 1. Changes in body weight

\begin{tabular}{cccc}
\hline Dose $(\mathrm{Gy})$ & No. of rats & Preirradiation $(\mathrm{g})$ & 2 wk after irradiation $(\mathrm{g})$ \\
\hline 0 & 2 & $267.88 \pm 3.34$ & $324.24 \pm 2.33$ \\
14 & 4 & $264.14 \pm 10.21$ & $347.96 \pm 20.78$ \\
16 & 4 & $270.29 \pm 7.24$ & $356.25 \pm 11.03$ \\
18 & 4 & $269.61 \pm 7.84$ & $357.41 \pm 25.79$ \\
20 & 4 & $266.20 \pm 15.12$ & $341.55 \pm 32.23$ \\
\hline
\end{tabular}

Values are presented as mean \pm standard deviation.

Mi Hyun Seo et al: Development of a standardized mucositis and osteoradionecrosis animal model using external radiation. JKorean Assoc Oral Maxillofac Surg 2020 
were designated as regions of interest (ROIs). Each ROI was defined as a volume of 50 pixels $\times 130$ pixels $\times 132$ pixels. Bone quality and volume were evaluated using Micro-CT image. Root rests within the ROI were excepted. Equivalent thresholds were applied for every image to divide mineralized bone from the background. Within each ROI, bone mineral density (BMD, \%), bone volume $\left(\mathrm{BV}, \mathrm{mm}^{2}\right)$, tissue volume $\left(\mathrm{TV}, \mathrm{mm}^{2}\right)$, and BV/TV, trabecular spaces (Tb.Sp., mm) were measured and compared.

\section{Histological evaluation}

After micro-CT scanning, we resected and decalcified the surgical fields at the mandible using $0.5 \mathrm{M}$ ethylenediaminetetraacetic acid (EDTA) solution ( $\mathrm{pH} 8.0)$ for three to four days, with replacement of the acid solution on the second day. The specimens were then dehydrated in $70 \%$ ethanol, fixed in $10 \%$ formalin-buffered solution, and embedded in paraffin wax.(Fig. 1. C) For histological staining, decalcified paraffin sections were cleaned with xylene for 10 minutes, and 5- $\mu \mathrm{m}$ thick slices were prepared and then stained with $\mathrm{H} \& \mathrm{E}$. The interesting region was designated as that in the level of extraction of the first molar.(Fig. 1. C) The slides were examined using a BX41 Light Microscope (Olympus, Tokyo, Japan). The numbers of osteoclasts and osteoblasts were counted at a high-power field $(\mathrm{HPF}, \times 200)$. Three HPFs were examined within the area of the extraction socket and the numbers of osteoclasts and osteoblasts were counted. The mean values were calculated and used in the statistical analysis.

\section{Statistics}

Statistical analyses were descriptive and done with the
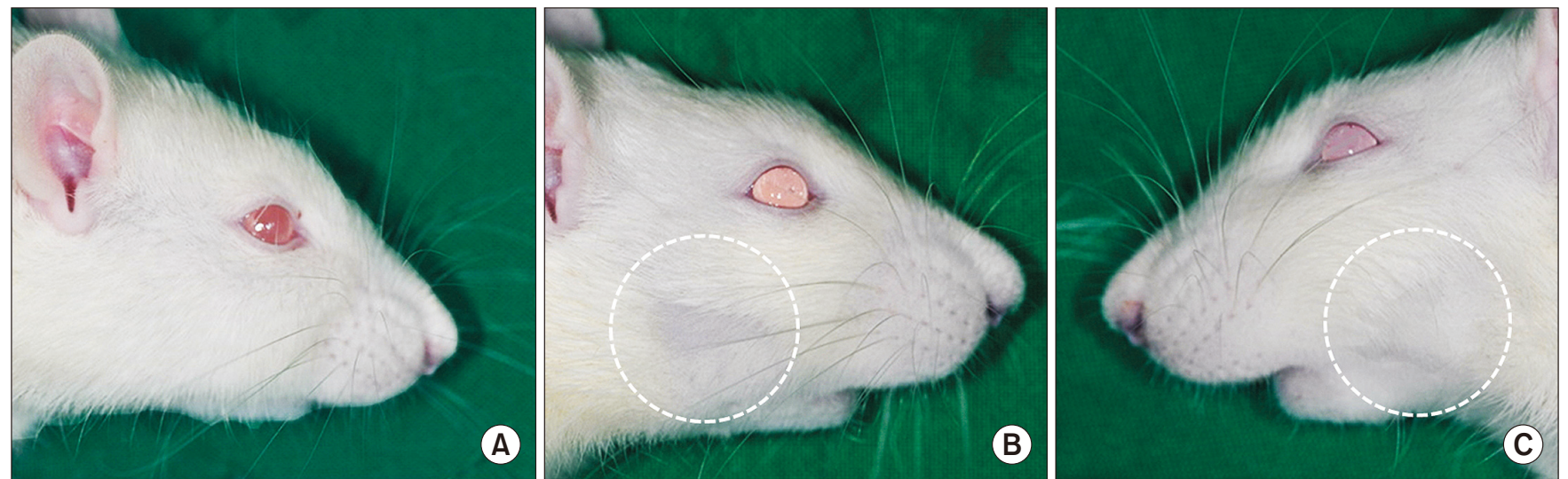

Fig. 2. Progression of hair loss in the clinical photo. A. Control group. B. Two weeks after irradiation. C. Two weeks after irradiation (contralateral side).

Mi Hyun Seo et al: Development of a standardized mucositis and osteoradionecrosis animal model using external radiation. J Korean Assoc Oral Maxillofac Surg 2020
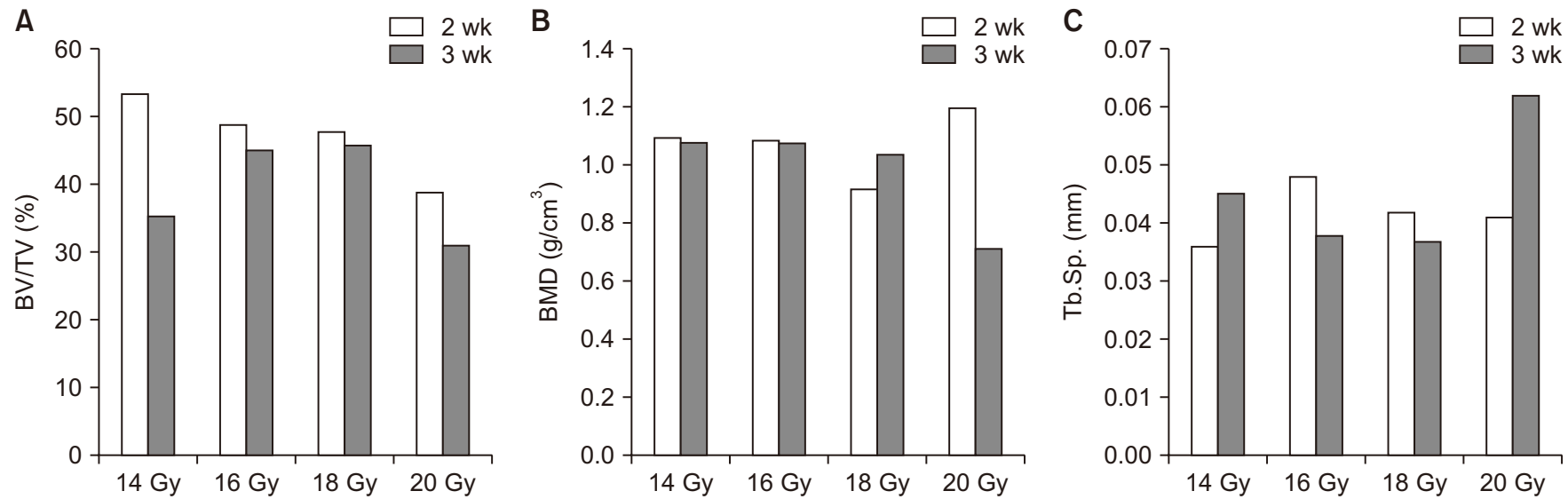

Fig. 3. Differences in bone volume/tissue volume (BV/TV) (A), bone mineral densities (BMD) (B), and trabecular spaces (Tb.Sp.) (C) between radiation doses and observation period.

Mi Hyun Seo et al: Development of a standardized mucositis and osteoradionecrosis animal model using external radiation. J Korean Assoc Oral Maxillofac Surg 2020 

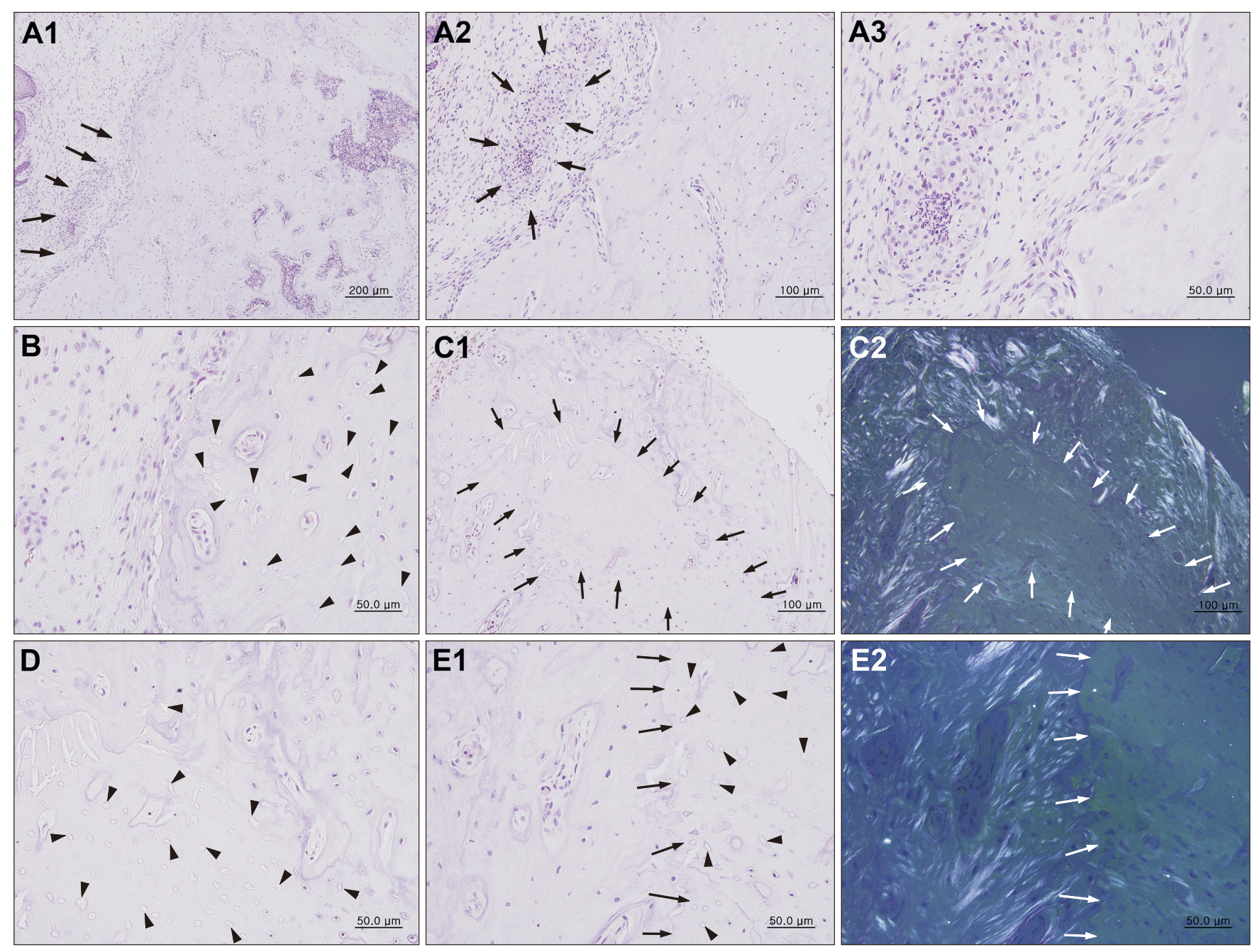

Fig. 4. Histological findings of H\&E staining of the jaw bone irradiated with 16 Gy showing resorption line (A1; arrows), microabscess (A2; arrows, A3), and dead bone (C1, C2, E1, E2; arrows), with empty osteocyte lacunae (B, D, E1; arrowheads) in the defect site. A1 arrows: resorption line; A2 arrow, A3: abscess border line and abscess area; B arrowheads: empty osteocyte lacunae; C1, C2 arrows: dead bone borderline; D, E1 arrowheads: empty osteocyte lacunae; E1, E2 arrows: dead bone borderline. (A1: ×100; A2: ×200; A3, B: ×400; C1, C2: $\times 200$ and polarized view in C2; D, E1, E2: ×1,000 and polarized view in E2)

Mi Hyun Seo et al: Development of a standardized mucositis and osteoradionecrosis animal model using external radiation. J Korean Assoc Oral Maxillofac Surg 2020

IBM SPSS Statistics program (ver. 22.0; IBM, Armonk, NY, USA). The differences in the osteoblast/HPF and osteoclast/ HPF between the groups were analyzed statistically using ANOVA method, and the Bonferroni method was used for post-test. Results were considered statistically significant at $P<0.05$

\section{Results}

\section{Clinical evaluation}

In all groups, after an average of two weeks of follow-up, radiation-induced side effects such as localized hair loss in the right and left mandible were noticeable. The progression of the skin alopecia was different depending on the irradiation dose; specifically, it could be found two or three weeks after 16 Gy (group 2), 18 Gy (group 3), and 20 Gy (group 4) of irradiation.(Fig. 2) The length of the hair shaft was short and hair loss was serious. Exposure of the epidermis, erythema, and ulcers were absent. Moreover, there were no differences between presentation two weeks and three weeks after irradiation in the same radiation dosage group. We did also detect changes in the body weight of the rats after irradiation. (Table 1)

\section{Micro-CT analysis}

No significant difference was found between the parame- 
ters, such as BV, BV/TV, BMD, and Tb.Sp., depending on the dose of radiation and the time of follow-up. A standardized method of quantification was achieved in all samples. However, BV/TV showed a tendency to decrease with increasing irradiation dose at 2 weeks samples, and BV/TV was slightly lower in 3 weeks than 2 weeks. BMD shows decreased at 20 Gy in the 3 weeks sample. Tb.Sp. was increased at 20 Gy in the 3 weeks sample.(Fig. 3)

\section{Histologic findings}

Morphological changes of the right mandible at the coronal level of first molar extraction site were detected. In the
H\&E staining sections, an increased number of osteoclast and inflammation cells at two weeks after 14 Gy irradiation. Additionally, fibrosis could be observed in the same sections at three weeks after 14 Gy irradiation. In group 2 (16 Gy), increased fibrosis was not present at two and three weeks after irradiation and resorption lines with microabscess and dead bone were found.(Fig. 4)

Either increased osteoclast or fibrosis as well as the presence of necrotic bone could be observed in groups 3 and 4 . Increased fibrosis and decreased BV were observed in these two groups at three weeks after irradiation as compared with at two weeks. Histological findings with 18 Gy irradiation showing more dead bone than that seen with 16 Gy dose and
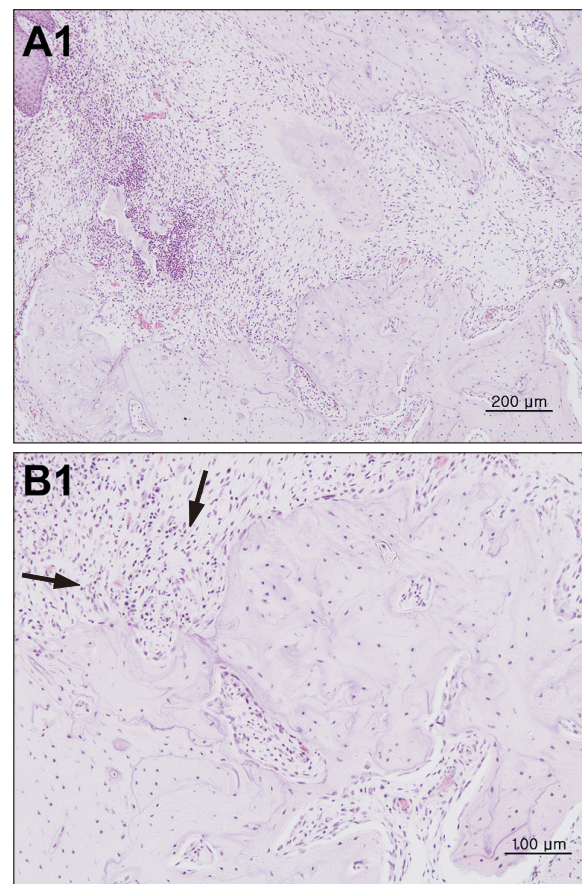

\section{C1}

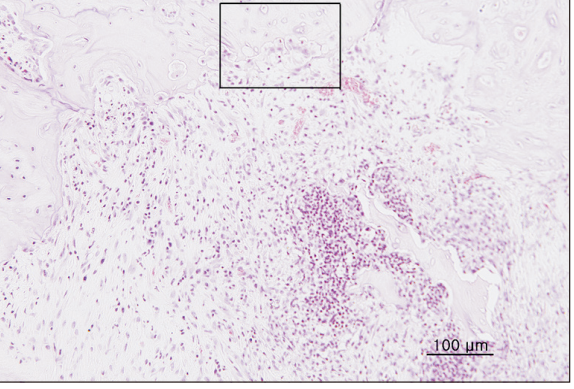

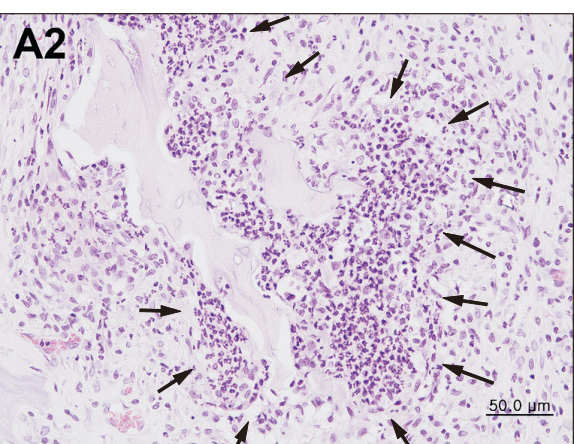
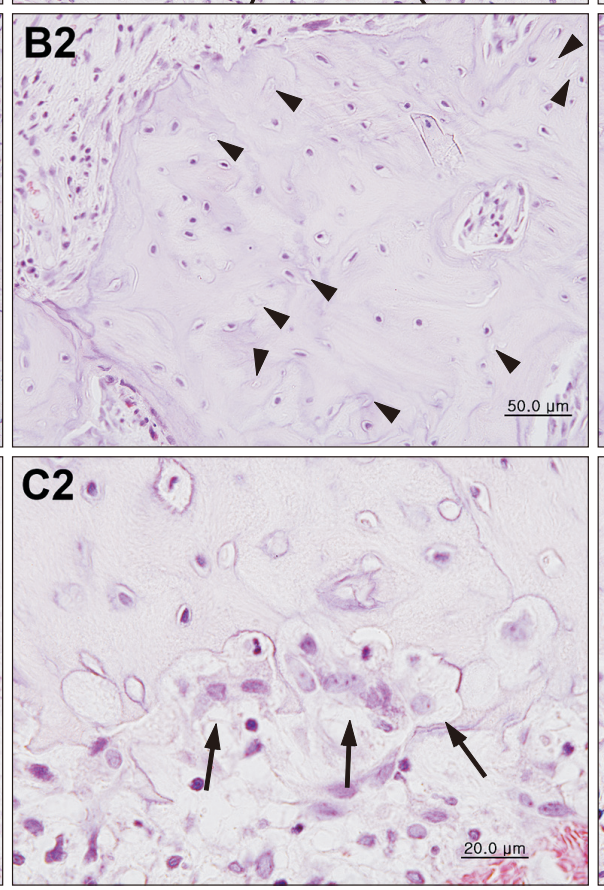
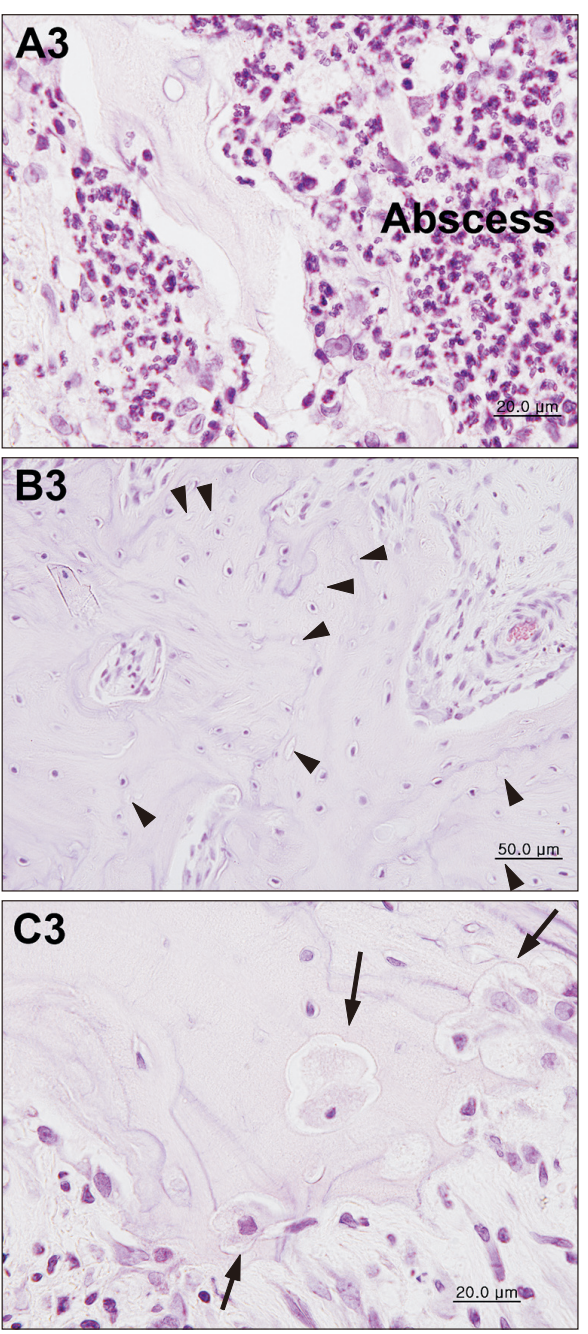

Fig. 5. Histological findings of H\&E staining of the jaw bone irradiated with 18 Gy showing more dead bone than that seen with 16 Gy dose and the presence of resorption with osteoclast cells. Abscesses (A1, A2; arrows, A3), inflammatory infiltrate (B1; arrows), and empty osteocyte lacunae (B2, B3; arrowheads) were observed. The close-up of the box in C1 is C2. There are numerous osteoclast cells observed in the radionecrotic lesion borderlines. A1, A2 arrows, A3: abscess and abscess borderline; B1 arrows: inflammatory cell; B2, B3 arrowheads: empty osteocyte lacunae; C1, C2 arrows: osteoclast cells; C3 arrows: internal osteoclast cells. (A1: $\times 100$; A2: $\times 400$, A3: $\times 1,000, \mathrm{~B} 1: \times 200, \mathrm{~B} 2, \mathrm{~B} 3: \times 400 ; \mathrm{C} 1: \times 200 ; \mathrm{C} 2, \mathrm{C} 3: \times 1,000)$

Mi Hyun Seo et al: Development of a standardized mucositis and osteoradionecrosis animal model using external radiation. J Korean Assoc Oral Maxillofac Surg 2020 
the presence of resorption with osteoclast cells. There are numerous osteoclast cells observed in the radionecrotic lesion borderlines.(Fig. 5) The histological features with 20 Gy irradiation showed the presence of resorption line with osteoclast cells, inflammatory infiltrate and empty osteocyte lacunae. (Fig. 6) These results may explain the progression of ORN along with that of inflammation, fibrosis, and bone resorption.

There were increases in the number of osteoclasts and fibrosis, decreases in the number of osteoblasts, and the presence of bony space in the radiated samples. Notably, as the radiation dose increase, the number of osteoblasts tended to decrease and the number of osteoclasts tended to increase. (Table 2) There was statistically significant difference in osteoblast/HPF between 14 Gy and 18 and 20 Gy $(P<0.05)$.
There was a significant difference in osteoclast/HPF between 14 Gy and 16, 18, and $20 \mathrm{~Gy}$, and there was a significant difference between $16 \mathrm{~Gy}$ and 18 and $20 \mathrm{~Gy}(P<0.05)$.(Table 3) These results suggest that osteoblasts were affected by

Table 2. Cell counts of osteoblasts and osteoclasts following irradiation

\begin{tabular}{crrrrr}
\hline \multirow{2}{*}{ Dose $(\mathrm{Gy})$} & \multicolumn{2}{c}{ Osteoblast/HPF } & & \multicolumn{2}{c}{ Osteoclast/HPF } \\
\cline { 2 - 3 } \cline { 5 - 6 } & \multicolumn{1}{c}{$2 \mathrm{wk}$} & $3 \mathrm{wk}$ & & $2 \mathrm{wk}$ & $3 \mathrm{wk}$ \\
\hline 14 & $11.0 \pm 1.0$ & $5.5 \pm 0.5$ & & $1.0 \pm 0.0$ & $0.5 \pm 0.5$ \\
16 & $6.0 \pm 1.0$ & $5.0 \pm 3.0$ & & $7.5 \pm 1.5$ & $9.0 \pm 1.0$ \\
18 & $2.5 \pm 1.5$ & $0.5 \pm 0.5$ & & $10.5 \pm 1.5$ & $9.5 \pm 4.5$ \\
20 & $1.5 \pm 0.5$ & $0.5 \pm 0.5$ & & $19.0 \pm 1.0$ & $19.5 \pm 3.5$ \\
\hline
\end{tabular}

(HPF: high-power field)

Values are presented as mean \pm standard deviation.

Mi Hyun Seo et al: Development of a standardized mucositis and osteoradionecrosis animal model using external radiation. J Korean Assoc Oral Maxillofac Surg 2020
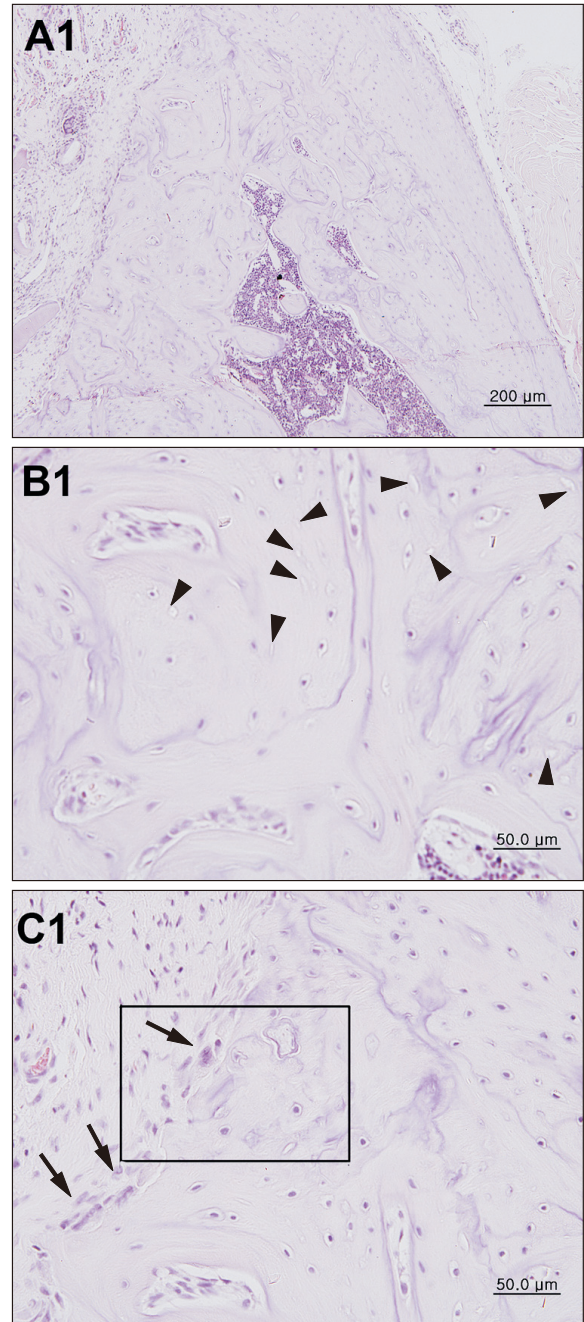
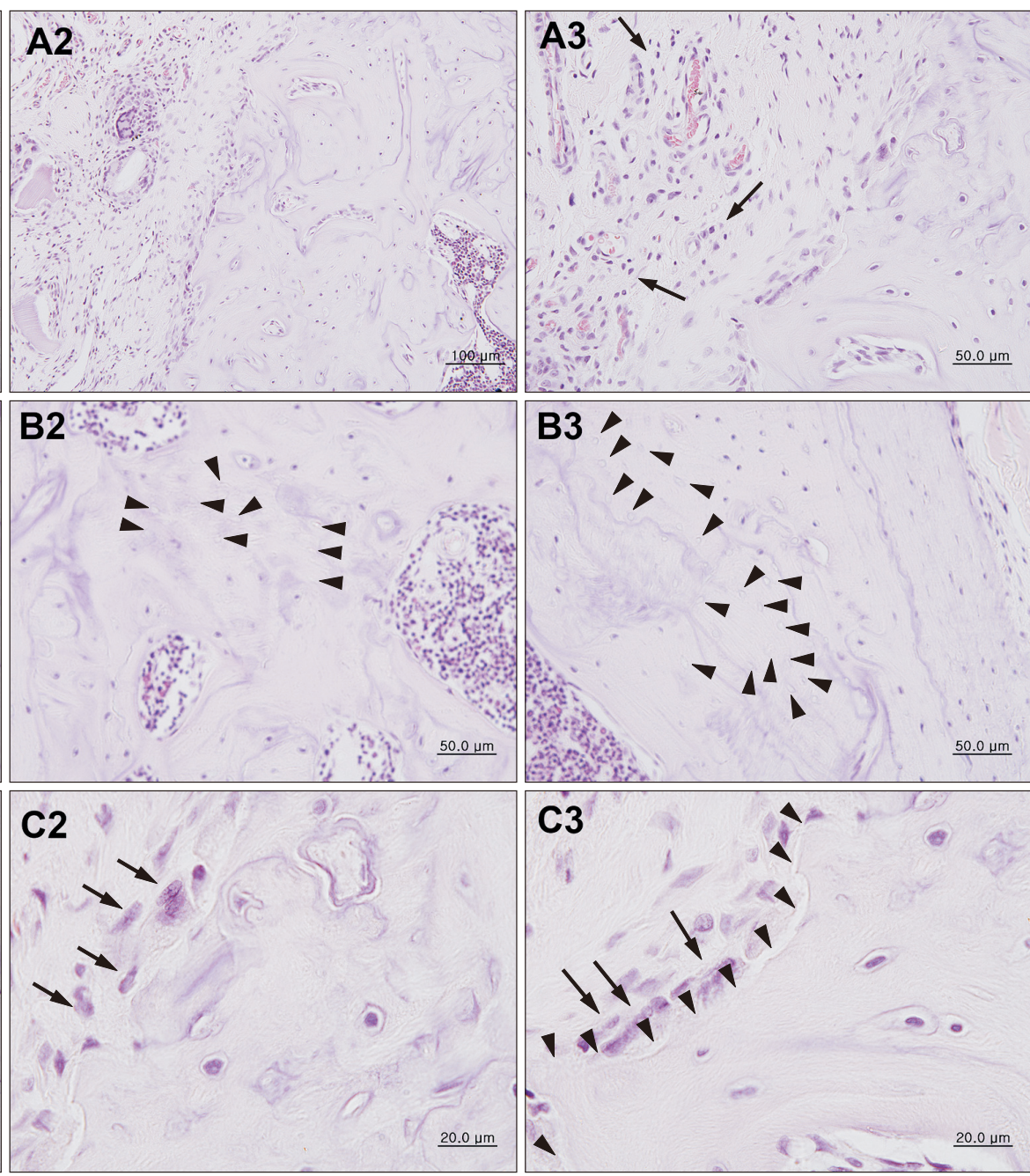

Fig. 6. Histological findings of H\&E staining of the jaw bone irradiated with 20 Gy. The histological features with 20 Gy irradiation showed the presence of resorption line (A1, A2) with osteoclast cells (C1, C2, C3; arrows), inflammatory infiltrate (A3) and empty osteocyte lacunae (B1, B2, B3; arrowheads). A1, A2: resorption line; A3 arrows: inflammatory cell; B1, B2, B3 arrowheads: empty osteocyte lacunae; C1, C2, C3 arrows: osteoclast cells; C3 arrowheads: dead bone border line. (A1: ×100; A2: ×200, A3, B1, B2, B3, C1: ×400; C2, C3: ×1,000) Mi Hyun Seo et al: Development of a standardized mucositis and osteoradionecrosis animal model using external radiation. J Korean Assoc Oral Maxillofac Surg 2020 
Table 3. Multiple comparisons using ANOVA

\begin{tabular}{|c|c|c|c|c|c|c|}
\hline & \multirow{2}{*}{ (I) dose (Gy) } & \multirow{2}{*}{ (J) dose (Gy) } & \multirow{2}{*}{$\begin{array}{l}\text { Mean differences } \\
(\mathrm{I}-\mathrm{J})\end{array}$} & \multirow{2}{*}{$P$-value } & \multicolumn{2}{|c|}{$95 \% \mathrm{CI}$} \\
\hline & & & & & Lower & Upper \\
\hline \multirow{12}{*}{ Osteoblast/HPF } & 14 & 16 & 2.75 & 0.720 & -2.4284 & 7.9284 \\
\hline & & 18 & $6.75^{*}$ & 0.009 & 1.5716 & 11.9284 \\
\hline & & 20 & $7.25 *$ & 0.005 & 2.0716 & 12.4284 \\
\hline & 16 & 14 & -2.75 & 0.720 & -7.9284 & 2.4284 \\
\hline & & 18 & 4.00 & 0.189 & -1.1784 & 9.1784 \\
\hline & & 20 & $4.50 *$ & 0.018 & -0.6784 & 9.6784 \\
\hline & 18 & 14 & $-6.75 *$ & 0.009 & -11.9284 & -1.5716 \\
\hline & & 16 & -4.00 & 0.189 & -1.1784 & 9.1784 \\
\hline & & 20 & 0.50 & 1.000 & -4.6784 & 5.6784 \\
\hline & 20 & 14 & $-7.25^{*}$ & 0.005 & -12.4284 & -2.0716 \\
\hline & & 16 & -4.50 & 0.108 & -9.6784 & 0.6784 \\
\hline & & 18 & -0.50 & 1.000 & -5.6784 & 4.6784 \\
\hline \multirow[t]{12}{*}{ Osteoclast/HPF } & 14 & 16 & $-7.50^{*}$ & 0.010 & -13.3364 & -1.6636 \\
\hline & & 18 & $-9.25 *$ & 0.002 & -15.0564 & -3.4136 \\
\hline & & 20 & $-18.50 *$ & 0.000 & -16.8364 & -5.1636 \\
\hline & 16 & 14 & $-7.50 *$ & 0.010 & 1.6636 & 13.3364 \\
\hline & & 18 & -1.75 & 1.000 & -7.5864 & 4.0864 \\
\hline & & 20 & $-11.00^{*}$ & 0.000 & -16.8364 & -5.1636 \\
\hline & 18 & 14 & $9.25^{*}$ & 0.002 & 3.4136 & 15.0864 \\
\hline & & 16 & 1.75 & 1.000 & -4.0864 & 7.5864 \\
\hline & & 20 & $-9.25^{*}$ & 0.002 & -15.0864 & -3.4136 \\
\hline & 20 & 14 & $18.50^{*}$ & 0.000 & 12.6636 & 24.3364 \\
\hline & & 16 & $11.00 *$ & 0.000 & 5.1636 & 16.8364 \\
\hline & & 18 & $9.25 *$ & 0.002 & 3.4136 & 15.0864 \\
\hline
\end{tabular}

(CI: confidence interval)

$* P<0.05$.

Mi Hyun Seo et al: Development of a standardized mucositis and osteoradionecrosis animal model using external radiation. J Korean Assoc Oral Maxillofac Surg 2020

radiation and degraded more than 18 Gy. Changes of bone resorption can be affected by radiation dose increment more sensitively.

\section{Discussion}

In this study, the appearance of skin alopecia varied depending on the irradiation dose. However, there were no differences between two weeks and three weeks after irradiation. In the histological sections, increased fibrosis, inflammation, and osteoclast number in all radiated groups at the two different observation periods was seen. Observation also revealed decreased normal bone in the 18 Gy and 20 Gy groups at three weeks after irradiation as compared with at two weeks. The CT parameters showed no significant differences among the groups.

The experimental design we planned according to the previous studies described by Kurihashi et al. ${ }^{18}$ demonstrated that the performance of tooth extraction at one week after radiation therapy is the optimal period for ORN occurrence. Ryu et al. ${ }^{17}$ reported that histological damage was healed at 14 days after 14 Gy and 16 Gy of radiation in a mouse model, while death occurred at nine days and 10 days after 18 Gy and 20 Gy of radiation. A single 20 Gy dose of irradiation is appropriate to maximize the reduction in bone regeneration and minimize animal morbidity according to Niehoff et al. ${ }^{19}$. Tamplen et al. ${ }^{20}$ described that the early manifestations of ORN appeared beginning at 21 days after irradiation. In the present study, we exposed the extraction region to a single dose of $14,16,18$, or 20 Gy of radiation at one week following first molar extraction.

Exposure to radiation damages the DNA of the various cells of the skin and controlled cell growth. In addition, alopecia can occur, and is caused by an insufficient supply of nutrients due to the reduction of blood vessels in the dermis layer. Following a dose of irradiation above 3 Gy, hair loss may be observed (at two to three weeks after irradiation); above $6 \mathrm{~Gy}$, erythema may arise; and at 8 to $15 \mathrm{~Gy}$, dry desquamation occurs, while, at 15 to $20 \mathrm{~Gy}$, moist desquamation occurs (at three to four weeks after irradiation) ${ }^{21}$. In our study, we only observed alopecia in different degrees and did not see erythema or ulceration. Moreover, there were no differences between at two weeks and at three weeks after irradiation.

In a previous experimental study, the thickness of the epithelium of the tongue was reduced after irradiation in a mouse mode ${ }^{17}$. Histopathologic analysis of radiation therapy shows chronic inflammation and necrotic bone. Arnold et 
al. ${ }^{16}$ developed a rabbit mandibular ORN model. After administering radiation doses of $6.5,7.0,7.5,8.0,8.5$, and 9.0 Gy, the histological sections showed immature woven bones in the high-dose groups (i.e., 8.5 Gy and 9.0 Gy). Tamplen et $\mathrm{al}^{20}$ demonstrated that osteoclasts experienced a significant increase and osteoblast experienced a decrease in the radiation group as compared with in the controls. Radiation induces depletion in the number of osteocytes and an increase in empty lacunae $\mathrm{e}^{22}$. In our histologic evaluations, we found increased fibrosis, inflammation, and osteoclast numbers in all radiated groups at two and three weeks after irradiation. The number of osteoblasts in the radiated groups were significant decreased versus in the control group. The mandible is a highly cortical membranous bone with little blood supply. In a rabbit model ${ }^{9}$, there was no significant difference found between the bone density of irradiated versus nonirradiated samples. In our study, BMD was measured using micro-CT and there was no significant difference between findings in different radiation dose groups; however, BV/TV values were decreased according to the increase of radiation doses. Radiation therapy-induced alterations will affect both remodeling capacity and biomechanical properties.

In the present study, local irradiation with a single dose of 14 Gy or 16 Gy was well-tolerated by the rats. However, the healing of bone defects after irradiation gets worse with the increase in dose and time of irradiation. The present study presents a well-established rat model that is comparable to current radiation treatment protocols for human head and neck cancer. A detailed and standardized animal model clinically similar to ORN of the jaw is a valuable tool that can be used to investigate the underlying pathophysiology of the disease and evaluate any potential treatment modalities.

\section{Conclusion}

Our simple but updated animal model is similar to the human mandibular ORN, allowing for subsequent radiological and histologic analysis of bone defects caused by irradiation. Further cell-specific staining, including immunohistochemistry, can be used with this model to study the potential cellular mechanisms of mandibular ORN and to trial any therapeutic options.

\section{ORCID}

Mi Hyun Seo, https://orcid.org/0000-0001-8220-6480

Min Young Lee, https://orcid.org/0000-0002-3462-989X

Mi Young Eo, https://orcid.org/0000-0001-7055-9924
Suk Keun Lee, https://orcid.org/0000-0001-6255-4197

Kyung Mi Woo, https://orcid.org/0000-0002-2782-1189

Soung Min Kim, https://orcid.org/0000-0002-6916-0489

\section{Authors' Contributions}

M.H.S. participated in data collection and wrote the manuscript. M.Y.L. participated in the experimental animal management. M.Y.E. participated in the study design and performed the experiment. S.K.L. participated in data analysis. K.M.W. and S.M.K. participated in the study design and coordination and helped to draft the manuscript. All authors read and approved the final manuscript.

\section{Acknowledgements}

We would like to thank to the Korean Institute of Radiological and Medical sciences, Laboratory Animal center for using X-RAD 320 irradiator, and this study was supported by a grant of the Korean Health Technology R\&D project, Ministry of Health \&Welfare, Republic of Korea (A120822 and HI15C0689).

\section{Ethics Approval and Consent to Participate}

The present study's protocol was reviewed and approved by the Seoul National University Institutional Animal Care and Use Committee (SNU-121123-12-11).

\section{Conflict of Interest}

No potential conflict of interest relevant to this article was reported.

\section{References}

1. Kim SM, Woo KM, Myoung H, Lee JH, Lee SK. Tissue engineering treatment in osteomyelitis of the jaws. Tissue Eng Regen Med 2015;12:11-26. https://doi.org/10.1007/s13770-013-0414-4

2. Støre G, Boysen M. Mandibular osteoradionecrosis: clinical behaviour and diagnostic aspects. Clin Otolaryngol Allied Sci 2000;25:378-84. https://doi.org/10.1046/j.1365-2273.2000.00367.x

3. Costa DA, Costa TP, Netto EC, Joaquim N, Ventura I, Pratas AC, et al. New perspectives on the conservative management of osteoradionecrosis of the mandible: a literature review. Head Neck 2016;38:1708-16. https://doi.org/10.1002/hed.24495

4. Marx RE. Osteoradionecrosis: a new concept of its pathophysiology. J Oral Maxillofac Surg 1983;41:283-8. https://doi. org/10.1016/0278-2391(83)90294-x

5. Delanian S, Lefaix JL. The radiation-induced fibroatrophic process: therapeutic perspective via the antioxidant pathway. Radiother On- 
col 2004;73:119-31. https://doi.org/10.1016/j.radonc.2004.08.021

6. Monson LA, Jing XL, Donneys A, Farberg AS, Buchman SR. Dose-response effect of human equivalent radiation in the mandible. J Craniofac Surg 2013;24:1593-8. https://doi.org/10.1097/ SCS.0b013e31826cfeea

7. Cohen M, Nishimura I, Tamplen M, Hokugo A, Beumer J, Steinberg ML, et al. Animal model of radiogenic bone damage to study mandibular osteoradionecrosis. Am J Otolaryngol 2011;32:291300. https://doi.org/10.1016/j.amjoto.2010.06.001

8. Vissink A, Burlage FR, Spijkervet FK, Jansma J, Coppes RP. Prevention and treatment of the consequences of head and neck radiotherapy. Crit Rev Oral Biol Med 2003;14:213-25. https://doi. org/10.1177/154411130301400306

9. Zhang WB, Zheng LW, Chua D, Cheung LK. Bone regeneration after radiotherapy in an animal model. J Oral Maxillofac Surg 2010;68:2802-9. https://doi.org/10.1016/j.joms.2010.04.024

10. He J, Qiu W, Zhang Z, Wang Z, Zhang X, He Y. Effects of irradiation on growth and differentiation-related gene expression in osteoblasts. J Craniofac Surg 2011;22:1635-40. https://doi.org/10.1097/ SCS.0b013e31822e5f66

11. Springer IN, Niehoff P, Açil Y, Marget M, Lange A, Warnke PH, et al. BMP-2 and bFGF in an irradiated bone model. J Craniomaxillofac Surg 2008;36:210-7. https://doi.org/10.1016/j.jcms.2007.09.001

12. Grimm G. [Animal experimental studies on the radiation injuries in the mandibles of full grown rabbits. I. Morphological and histochemical findings]. Dtsch Zahn Mund Kieferheilkd Zentralbl Gesamte 1969;53:307-35. German.

13. Grimm G. [Animal experimental studies on the pathogenesis of radiogenic bone injuries in the mandibles of adult rabbits. II. Histometric data]. Dtsch Zahn Mund Kieferheilkd Zentralbl Gesamte 1970;54:352-62. German.

14. Hamilton SA, Pecaut MJ, Gridley DS, Travis ND, Bandstra ER, Willey JS, et al. A murine model for bone loss from therapeutic and space-relevant sources of radiation. J Appl Physiol (1985) 2006;101:789-93. https://doi.org/10.1152/japplphysiol.01078.2005

15. Würzler KK, DeWeese TL, Sebald W, Reddi AH. Radiationinduced impairment of bone healing can be overcome by recombinant human bone morphogenetic protein-2. J Craniofac Surg 1998;9:131-7. https://doi.org/10.1097/00001665-199803000-00009

16. Arnold M, Stas P, Kummermehr J, Schultz-Hector S, Trott KR.
Radiation-induced impairment of bone healing in the rat femur: effects of radiation dose, sequence and interval between surgery and irradiation. Radiother Oncol 1998;48:259-65. https://doi. org/10.1016/s0167-8140(98)00039-5

17. Ryu SH, Moon SY, Choi EK, Kim JH, Ahn SD, Song SY, et al. Establishment of a single dose radiation model of oral mucositis in mice. J Korean Soc Ther Radiol Oncol 2008;26:257-62. https://doi. org/10.3857/jkstro.2008.26.4.257

18. Kurihashi T, Iwata H, Nasu M, Yosue T. Experimental study on wound healing of alveolar bone sockets in the rat maxilla after Xray irradiation. Odontology 2002;90:35-42. https://doi.org/10.1007/ s102660200005

19. Niehoff P, Springer IN, Açil Y, Lange A, Marget M, Roldán JC, et al. HDR brachytherapy irradiation of the jaw - as a new experimental model of radiogenic bone damage. J Craniomaxillofac Surg 2008;36:203-9. https://doi.org/10.1016/j.jcms.2008.01.003

20. Tamplen M, Trapp K, Nishimura I, Armin B, Steinberg M, Beumer $\mathrm{J}$, et al. Standardized analysis of mandibular osteoradionecrosis in a rat model. Otolaryngol Head Neck Surg 2011;145:404-10. https:// doi.org/10.1177/0194599811400576

21. Nanashima N, Ito K, Ishikawa T, Nakano M, Nakamura T. Damage of hair follicle stem cells and alteration of keratin expression in external radiation-induced acute alopecia. Int J Mol Med 2012;30:579-84. https://doi.org/10.3892/ijmm.2012.1018

22. Tchanque-Fossuo CN, Monson LA, Farberg AS, Donneys A, Zehtabzadeh AJ, Razdolsky ER, et al. Dose-response effect of human equivalent radiation in the murine mandible: part I. A histomorphometric assessment. Plast Reconstr Surg 2011;128:114-21. https://doi.org/10.1097/PRS.0b013e31821741d4

How to cite this article: Seo MH, Lee MY, Eo MY, Lee SK, Woo KM, Kim SM. Development of a standardized mucositis and osteoradionecrosis animal model using external radiation. J Korean Assoc Oral Maxillofac Surg 2020;46:240-249. https://doi.org/10.5125/ jkaoms.2020.46.4.240 